\title{
Generalized Hough Transform for Shape Matching
}

\author{
Cecilia Di Ruberto \\ Department of Mathematics and Computer Science \\ University of Cagliari \\ Via Ospedale 72, 09124 Cagliari, Italy
}

\begin{abstract}
In this paper we propose a novel approach towards shape matching for image retrieval. The system takes advantages of generalized Hough transform, as it works well in detecting arbitrary shapes even in the presence of gaps and in handling rotation, scaling and shift variations, and solves the heavy computational aspect by introducing a preliminary automatic selection of the appropriate contour points to consider in the matching phase. The numerical simulations and comparisons have confirmed the effectiveness and the efficiency of the method proposed.
\end{abstract}

\section{General Terms}

Pattern Recognition, Graphics and Imaging, Computer Vision

\section{Keywords}

Hough Transform, image retrieval, dominant point, polygonal approximation, shape matching.

\section{INTRODUCTION}

One of the important tasks in the field of computer vision and image retrieval is the detection of specific shapes in a contour image. Shape representation methods and descriptors extract effective and perceptually important shape features. In this context it is desirable to preserve important characteristics of shape and, at the same time, to be invariant to transformations like scale, rotation and translation. Also it is expected that descriptors have a robust behavior respect to noise affected shapes or distorted, defective or occluded shapes, as like acts the human behavior in such situations. In addiction extracted features have to be suitable for indexing and online retrieval, i.e. low complexity and computational cost, and performing well not only on certain type of shapes. These features can be extracted from either shape boundary information or internal regions content. Based on such difference we can discern into contour-based methods and region-based methods for features extraction. Because of their semantically rich nature, contours are the most commonly used shape descriptors, and various methods for representing the contours of bidimensional objects have been proposed, each achieves, more or less successfully, the most desiderable features of a proper representation, such as data compression, simplicity of coding and decoding, scaling and invariance under rigid motions. The visual information about a shape described by its contour is concentrated at the dominant points. A set of points is considered as a dominant set if it is possible to construct the contour from these points using some method of interpolation, the polygon is the simplest one. The dominant points are regarded as representative features for the object contours, because they reserve the significant features of the digitized curve of the images. One of the advantages of using dominant points to represent contours is the high data reduction and its immediate impact on the efficiency of the subsequent feature extraction and shape matching algorithms. This is the reason why we have used the dominant points to improve the performance of Hough transform, both in computational time and in matching viewpoint.

To address the problem of shape matching, as for the detection methods, which are invariant to rotations, shifts and scale changes of objects, the generalized Hough transform (GHT) [1] has been proposed so far. However, this method is not suitable for a practical use from the computing time viewpoint. Also, if the contours of the objects are corrupted by noise, its direct application becomes useless. To solve these problems, a fuzzy generalized Hough transform (FGHT) [8], [6], [10] has been proposed. It is based on a fuzzy concept included in the GHT in order to realize the robust detection of arbitrary shapes in a image corrupted by heavy noise and to handle rotation, scaling, shift variations and partial occlusions. However, the performance of the FGHT is strongly influenced by the contour points chosen in the matching process, as we have observed during a large experimentation. To solve all these problems we have combined the effective formulation of the GHT with a selection of the appropriate contour points, i.e. dominant points, useful to describe a shape in order to get a new method for shape matching in image retrieval. The effectiveness and the validity of the proposed approach have been confirmed by computer simulations on a database of 216 images.

The paper is organized as follows. In section 2 we give a brief overview of Hough transform and its extensions. In section 3 we analyze the dominant points role for shape matching. Section 4 describes the proposed shape matching framework and some computer simulations and comparisons. Finally, section 5 concludes the work and offers the outlook for the future.

\section{GENERALIZED HOUGH TRANSFORM}

Hough transform (HT) was originally proposed by Hough [7] and later refined [5] to detect analytically defined shapes like straight lines, circles, ellipses, etc. from binary images. Afterwards it has been extended to generalized Hough transform [1] in order to detect arbitrary shapes. Different problems of image analysis and computer vision, among which edge/line linking, boundary/motion detection, object recognition and shape analysis, have been addressed by using the HT and GHT. One of the basic advantages of HT is that it works even for the broken edges, i.e. it is very tolerant of gaps in the true object boundaries and it is relatively unaffected by noise.

HT (and GHT) maps shapes like lines, circles, ellipses etc. (and arbitrary shapes) from the image space to a parameter space in which the shapes are represented in terms of their parameter values $[1,5]$. Each point on a shape in the image space is transformed into a curve in the parameter space, and 
all the points belonging to a particular shape are transformed to a family of curves passing through a common point. A model of a particular shape, called R-table, is created: the table contains the relationship between the boundary points and orientation, and the Hough parameters [1]. So, at first an arbitrary reference point in the object is selected and the shape is defined in terms of the distance and the angle of lines from the boundary to this reference point. For all points of the boundary, a line to the reference point is drawn. Then the orientation of the boundary is computed and a note in the table of the distance and the direction from the boundary point to the reference point at a location in the table indexed by the boundary orientation is created. The Hough transform space is so defined in terms of the possible positions of the shape in the image [5]. All the points belonging to a particular shape are transformed to a family of curves passing through a common point. The point of intersection of this family of curves represents the set of parameters defining the shape. In general, a cluster is formed in the parameter space corresponding to a shape in the image space. Given a particular shape, the family will include all the objects obtainable by translation, rotation and scaling. The edges/lines or curves of general shapes can therefore be detected by separating out these clusters (peaks) in the parameter space. However, in real images the shapes are often distorted from their true parametric forms due to the presence of noise, digitization error and shape variations. Even if the input image is perfect, it is often very difficult to describe the shapes in terms of the perfect model shape. Therefore, it may be difficult to find out a single peak or a compact cluster in the parameter space. In other words, a single shape in an image may get broken into more than one local peak in the parameter space. Consequently, we have a decrease in the amplitude of the peaks in the parameter space and, then, a difficulty in their detection. To solve this problem, around each point on the perfect shape defined by the parametric form, a small region can be defined, and each pixel in this region, i.e. each pixel in the neighborhood of the perfect shape, contributes to the accumulator space. Thus, the accumulator values show a smooth transition from the higher to lower values so that the peaks can be identified unambiguously even for distorted shapes. Because of noise, discretisation of the image and accumulator, and factors inherent in the application itself, we sometimes want to allow a little tolerance in the fitting of lines or other shapes to the edge pixels. We can do this by allowing a feature point to vote not just for a sharp line or curve in the accumulator, but to cast fuzzy votes for nearby possibilities [8]. In other words, this votes not just for all lines or other shapes that pass through the feature point but also for those that pass close by.

\section{ON DOMINANT POINTS ROLE}

Dominant points are those points that have curvature extreme on the curve and they can suitably describe the curve for both visual perception and recognition [3]. Dominant points can be applied to produce a simplified representation of a shape contour for further processing. This representation simplifies the analysis of the images by providing a set of feature points containing almost complete information of a contour. One of the main advantages of a good representation of bidimensional shapes through dominant points is the high data reduction and its immediately efficiency in feature extraction and shape matching algorithms. The data reduction leads to a higher speed during execution; furthermore, a lower memory usage is required, with immediate benefits both for storage and for transmission. Another important aspect concerns a better quality of data because, although the reduction of points could suggest a loss of information, one must consider the choice of points of interest, which contain the main features of the curve, discarding the rest. Therefore, an approximated curve will be more suitable for classification and extraction of attributes than the original curve, and less affected by noise. It is well known also that these points play a dominant role in shape perception by humans.

There are many approaches developed for detecting dominant points. They can be classified into two main categories: corner detection approaches and polygonal approximation approaches. Corner detection approaches aim to detect potential significant points, but they cannot represent smooth curve appropriately. The performance of these detectors depends on the accuracy of the curvature evaluation [13], [15]. For polygonal approximation approaches, sequential, iterative and optimal algorithms are commonly used. Sequential approaches are simple and fast, but the results depend on the location of the point where they start the scanalong process and they can miss important features. The iterative approaches split and merge curves iteratively until they meet the preset allowances. Their results may be far from the optimal one if a poor initial segmentation is used [9], [11]. The optimal approaches tend to find the optimal polygonal approximation based on specified criteria and error bound constraints [16], [17], [18]. The idea is to approximate a given curve by an optimal polygon with the minimal number of line segments such that the approximation error between the original curve and the corresponding line segments is no more than a pre-specified tolerance. Local optimal methods are very fast but their results may be very far from the optimal one. However, an exhaustive search for the vertices of the optimal polygon from the given set of data points results in an exponential complexity. Luckily, there exist some heuristic approaches (genetic algorithms and tabu search) which can find solutions very close to the global optimal one in a relative short time. In [4] we have presented a method that combines the dominant point detection and the ant colony optimization search. The method is inspired by the ant colony search suggested in [18] but it results in a much more efficient and effective approximation algorithm. We have used this approach to detect the suitable set of contour points to consider in the matching phase.

\section{THE SHAPE MATCHING SYSTEM}

Although GHT is useful for shape analysis in occluded and noisy environments, it suffers from heavy computation and large memory requirement to handle the rotation and scale variations. For this reason, GHT is not useful directly for image retrieval. To solve such problems we have combined the FGHT with the selection of appropriate points set used for shape representation in order to address image retrieval by shape similarity. Let's describe the main steps of the whole recognition process.

The first step consists of an automatic selection of the most significant points useful to describe a particular shape. In [4] we have presented a novel method for dominant point detection. Since of the efficiency and the efficacy of such approximation algorithm, we have used this dominant point selection to get the set of points for the matching phase. We have applied the selection process on each image included in the database.

The second step consists of creating the model, the R-table, for the query shape starting from the previously selected points set. 
The third step is the voting phase. The voting is achieved by incrementing a region and not a single pixel in the accumulator space. This allows to handle local variations in the neighbourhood of the shape points.

The last step consists in detecting the maxima in the accumulator space and in ranking all the images according to the similarity with the query shape.

The proposed recognition system has been tested on a database of 216 images, shown in Figure 1. Our images database benchmark is a MPEG-7 subset commonly used in shape retrieval and contour matching problems. It consists of 18 classes of common images, each containing 12 items.

In table 1 we show the distribution of the images retrieved and found by the proposed approach. The first column reports the query shape for each class while the others represent the items ordered by similarity to the query shape (the first top ten shapes).

In table 2 we show the average accuracy and the average time (in sec.) in retrieving a shape of each class. In the first line of the table we present the performance of our approach. In the second and the third line we show the performance in retrieving a shape without the automatic selection step, i.e. all the contour points are used in the matching phase, and by using a uniform contour sampling, respectively. In both the cases the accuracy is lower than that obtained by using the selection step, confirming the advantage in choosing the appropriate points for the matching phase. Also, we have completed the whole retrieval process in a much shorter time.

Finally, we have compared the proposed system to some of the best considered contour matching methods [12], [14], [2]. In [12] a modification of the standard assignment problem is proposed in order to preserve the ordering of the points induced from the shapes contours in the correspondence problem.

The proposed approach has produced significantly improved correspondences. In [14] the first Ant Colony Optimization algorithm specifically aimed at solving the Quadratic Assignment Problem for shape correspondence has been proposed. The approach has yielded high quality correspondence results and is computationally efficient. In [2] the authors have proposed a novel descriptor, the shape context, to estimate the shape similarity and correspondence. They have also demonstrated the invariance to several common image transformations. When compared to these methods, our approach has demonstrated its superiority both in quality of matching and in time.

\section{CONCLUSIONS}

The aim of this paper was to investigate a novel approach towards shape indexing. The system we have proposed takes advantage of GHT, as it works well in detecting arbitrary shapes even in the presence of noise and in handling rotation, scaling and shift variations, and solves the heavy computational aspect characterizing the HT by introducing a preliminary automatic selection of the contour points to consider in the matching phase, which represents our main research contribution. As we can see from the numerical results, all the simulations based on using the GHT in shape matching present the best accuracy values, but they take a longer time. Finally, we want to stress that the best results are obtained by using the dominant point detection method proposed in [4]. The presented recognition system represents a right compromise in term of accuracy and time, confirming the effectiveness and the efficiency of the method proposed for shape

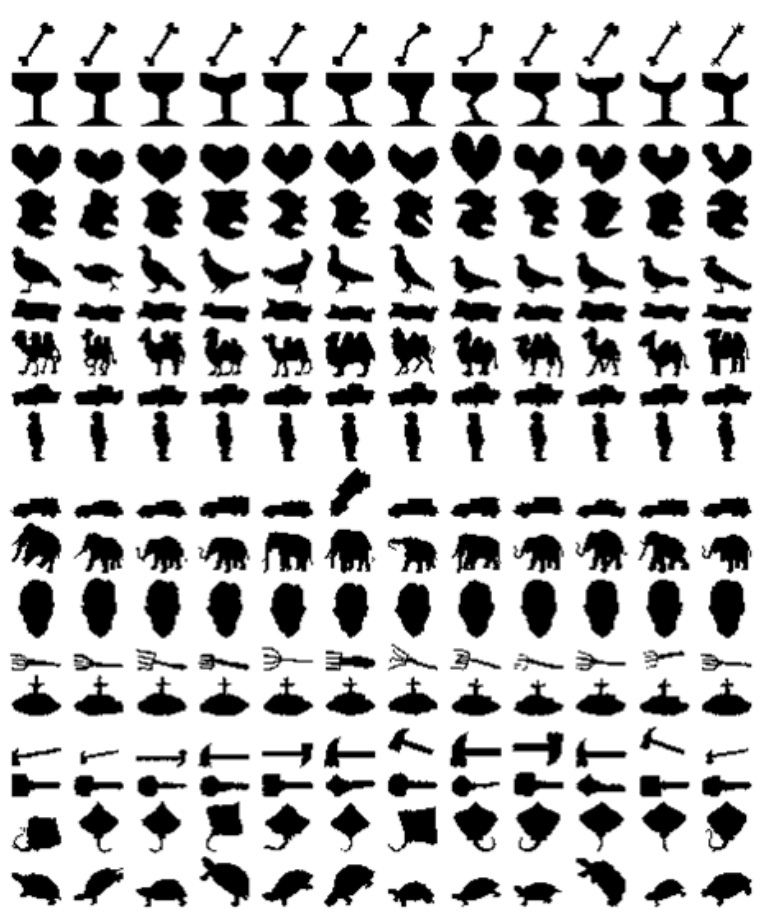

Figure1: Image database benchmark.

Matching. These initial experimental results are promising and encourage further experimentation on a much larger image database.

Table 1: A tabulation of the top ten similar shapes.

\begin{tabular}{|c|c|c|c|c|c|c|c|c|c|c|}
\hline Shape & \multicolumn{10}{|r|}{10} \\
\hline$\checkmark$ & $\checkmark$ & $\downarrow$ & $\downarrow$ & $\downarrow$ & $\checkmark$ & $\downarrow$ & $\downarrow$ & 1 & $I$ & $\downarrow$ \\
\hline$I$ & $I$ & $I$ & $I$ & $I$ & $I$ & 1 & $I$ & $I$ & $I$ & 1 \\
\hline$\bullet$ & $\varphi$ & ๑ & 0 & $v$ & $\bullet$ & $\bullet$ & $\varphi$ & $v$ & $v$ & $\varphi$ \\
\hline 8 & 8 & 2 & 8 & 8 & 5 & 8 & 8 & 8 & 3 & 8 \\
\hline$\lambda$ & h & 4 & $\lambda$ & $\downarrow$ & 1 & 1. & $\bullet$ & 1 & 1 & 1 \\
\hline$\approx$ & $=$ & - & $=$ & $=$ & 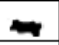 & $\sim$ & $=$ & $m$ & $m$ & 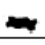 \\
\hline$y$ & in & (n) & X & in & 4 & 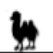 & 4 & 传 & $y$ & 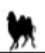 \\
\hline 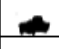 & 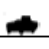 & 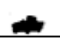 & 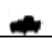 & $=$ & $\Rightarrow$ & 4 & - & $\Rightarrow$ & 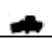 & - \\
\hline 1 & 1 & 1 & 1 & 1 & 1 & 1 & 1 & 1 & 1 & 1 \\
\hline- & - & - & $\Rightarrow$ & $\Rightarrow$ & - & - & $\Rightarrow$ & $\Rightarrow$ & - & $=$ \\
\hline m & m & n & m & an & 9 & (n) & A & Im & T & in \\
\hline 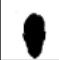 & 0 & 0 & 0 & 0 & 0 & 0 & 0 & $\theta$ & 0 & 0 \\
\hline 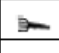 & $9-$ & 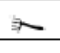 & 2 & 㕛 & 2 & 2 & 2 & 3 & 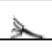 & $\underline{~}$ \\
\hline 5 & 5 & \pm & \pm & $b$ & \pm & 5 & \pm & 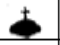 & \pm & \pm \\
\hline$\rightarrow$ & $\vdash$ & $\vdash$ & $\rightarrow$ & $\rightarrow$ & $r$ & $\vdash$ & $\vdash$ & $r$ & $F$ & $\leftarrow$ \\
\hline$\leftarrow$ & - & $\leftarrow$ & $\sigma$ & $\leftarrow$ & $\leftarrow$ & 1 & 上 & $\leftarrow$ & $\leftarrow$ & - \\
\hline $\boldsymbol{\mu}$ & $\eta$ & $\lambda$ & 5 & $\rightarrow$ & $\bullet$ & 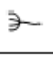 & 2 & 2 & $\vdash$ & $7-$ \\
\hline$a$ & 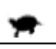 & $a$ & 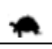 & 7 & - & 4 & 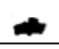 & 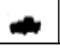 & 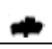 & 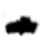 \\
\hline
\end{tabular}


Table 2: Numerical results (for details see the text).

\begin{tabular}{|c|c|c|}
\hline Method & accuracy & Time \\
\hline Our approach & 0.8981 & 0.29 \\
No selection step & 0.8704 & 8.07 \\
Uniform sampling & 0.8750 & 0.42 \\
Scott et alt. [12] & 0.8333 & 0.21 \\
Van Kaick et alt. [14] & 0.8426 & 0.62 \\
Belongie et alt. [2] & 0.8333 & 0.24 \\
\hline
\end{tabular}

\section{REFERENCES}

[1] D.H. Ballard, "Generalizing the Hough transform to detect arbitrary shapes", Pattern Recognition, 13(2): 111$-122(1981)$

[2] S. Belongie, J. Malik, J. Puzicha, "Shape matching and object recognition using shape contexts", IEEE Transactions on Pattern Analysis and Machine, 24(24):509--522 (2002).

[3] A. Carmona-Poyato, N.L. Fernandez-Garcia, R. MedinaCarnicer, F.J. Madrid-Cuevas, "Dominant point detection: A new proposal", Image and Vision Computing, 23:1226-1236 (2005).

[4] C. Di Ruberto, A. Morgera, "A new algorithm for polygonal approximation based on Ant Colony System", Lecture Notes in Computer Science, 5716:633--641 (2009)

[5] R.O. Duda, P.E. Hart, "Use of Hough transform to detect lines and curves in pictures", Commun. ACM., 15:11-15 (1972).

[6] J.H. Han, L.T.Koczy, T. Poston, "Hough transform", Pattern Recognition Letters", 15:649--658 (1994)Brown, L. D., Hua, H., and Gao, C. 2003. A widget framework for augmented interaction in SCAPE.

[7] P.V.C. Hough, "Method and means for recognizing complex patterns", US Patent, 3069654, (1962).
[8] H. Izadinia, F. Sadeghi, M.M. Ebadzadeh, "Fuzzy generalized Hough transform invariant to rotation and scale in noisy environment", FUZZ-IEEE 2009, Korea, August 20-24, 153--158 (2009).

[9] M. Marji, P. Siy, "A new algorithm for dominant points detection and polygonization of digital curves", Pattern Recognition, 36:2239-2251 (2003).

[10] K.P. Philip, E.L. Dove, D.D. McPherson, N.L. Gotteiner, W., Stanford, K.B. Chandran, "The fuzzy Hough transform-feature extraction in medical images", IEEE Trans. on Med. Imaging, 13:235--240 (1994).

[11] B.K. Ray, K.S. Ray, "Detection of significant points and polygonal approximation of digitized curves", Pattern Recognition Letters, 22:443--452 (1992).

[12] C. Scott, R. Nowak, "Robust contour matching via the order preserving assignment", IEEE Trans. on Image Processing, 15(7):1831--1838 (2006).

[13] C.H. Teh, R.T. Chin, "On the detection of dominant points on digital curves", IEEE Trans. on Pattern Analysis and Machine Intelligence, 11(8):859--871 (1989).

[14] O. van Kaick, G. Hamarneh, H. Zhang, P. Wighton, "Contour correspondence via ant colony optimization", 15th Pacific Conference on Computer Graphics and Applications, 271--280 (2007).

[15] W.Y. Wu, "Dominant point detection using adaptive bending value", Image and Vision Computing, 21:517-525 (2003).

[16] P.Y. Yin, "Genetic algorithms for polygonal approximation of digital curves", Int. J. Pattern Recognition Artif. Intell., 13:1--22 (1999).

[17] P.Y. Yin, "A tabu search approach to the polygonal approximation of digital curves", Int. J. Pattern Recognition Artif. Intell., 14:243--255 (2000).

[18] P.Y. Yin, "Ant colony search algorithms for optimal polygonal approximation of plane curves", Pattern Recognition, 36(8):1783--1797 (2003). 